\title{
Incidence of Sunitinib induced thyroid dysfunction in renal cell carcinoma - a pilot retrospective audit
}

\section{S. Zhyzhneuskaya, L.Erete, J.Czechowska, W.Anderson, A Humphreys, S.Nag The James Cook University Hospital, Middlesbrough, TS4 3BW}

South Tees Hospitals $\mathbf{N H}$ NHS Foundation Trust

\section{Introduction}

Tyrosine kinase inhibitors (TKI) are an emerging group of anti-growth factor agents used in the treatment of solid cancers. Treatment is associated thyroid dysfunction. Sunitinib is licensed for the treatment of metastatic renal cell carcinoma.

\section{Objective}

To determine the incidence of Sunitinib induced thyroid dysfunction and its management in patients with renal cell carcinoma (RCC).

\section{Methods}

Retrospective case note analysis of patients started on Sunitinib for metastatic RCC between January 2010 and December 2012 at the Oncology Unit in The James Cook University Hospital.

Results $>>>>>>>>>>>>>>>>>>>>>>>>>>>>$

31 patients were started on Sunitinib between 2010-2012. One patient had pre-existing Primary Hypothyroidism and was excluded from analysis. Baseline thyroid function tests (TFT) were done in $93 \%$ of patients. The majority of patients $(n=26)$ were euthyroid pre-treatment; at baseline, 3 patients $(10 \%)$ had subclinical hypothyroidism and 1 patient (3.3\%) had subclinical hyperthyroidism.

Mean duration of follow up was 53.33 weeks. Mean interval between starting Sunitinib and 1st TFT check was 3.3 weeks (range 2-12 weeks, SD 2.28). Mean interval to developing abnormal TSH was 9.3 weeks (range 2-42 weeks, SD 11.58). Primary

hypothyroidism in this cohort developed at 27.7 weeks (range- 4-46 weeks). The mean time to commencing Levothyroxine (LT4) therapy was 55.5 weeks (range 21-105 weeks).

Sunitinib induced hypothyroidism developed in 6 patients $(20 \%)$ whilst subclinical hypothyroidism developed in 2 patients (6.6\%). 21 patients $(70 \%)$ were biochemically euthyroid. Thyroid status of one patient with baseline subclinical hyperthyroidism remained unchanged. 4 patients (13.3\%) developed transient subclinical hypothyroidism. Mean TSH level at start of LT4 therapy was $55.7 \mathrm{mIU} / \mathrm{l}$ (range 19.43-107.24) and mean Free T4 was $8.4 \mathrm{pmol} / \mathrm{l}$ (range 3.5-11.8). LT4 was commenced at a mean dose of $39 \mathrm{mcg}$ once daily (range $25-50 \mathrm{mcg}$ ) and the average final dose was 118 mcg once daily (range $50-225 \mathrm{mcg}$ ).

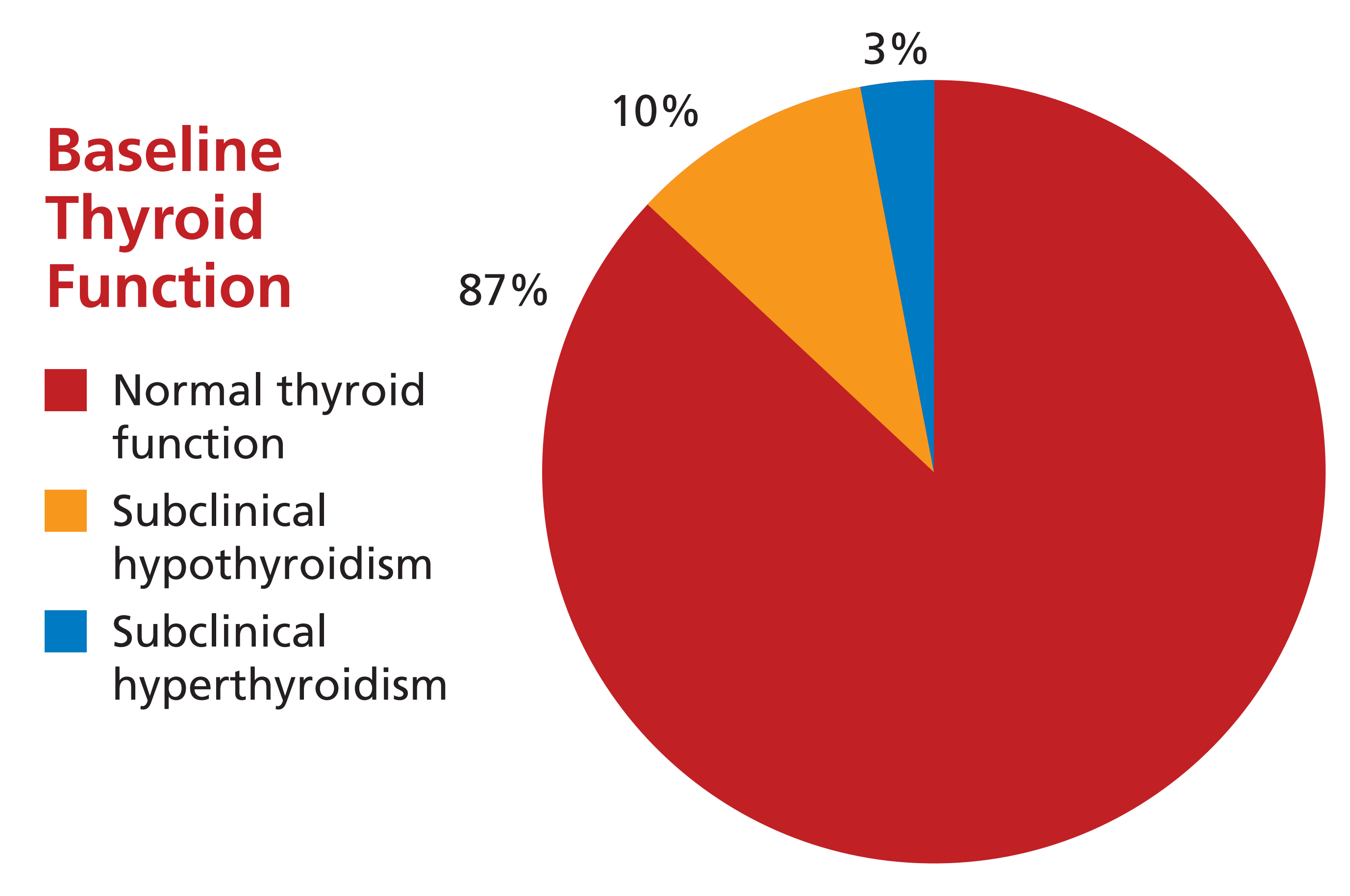

Summary of the outcomes

Euthyroid Hypothyroidism $20 \%$

Subclinical hypothyroidism Subclinical hyperthyroidism

Transient subclinical hypothyroidism: 4(13.3\%) Transient subclinical hyperthyroidism: 1(3.3\%)

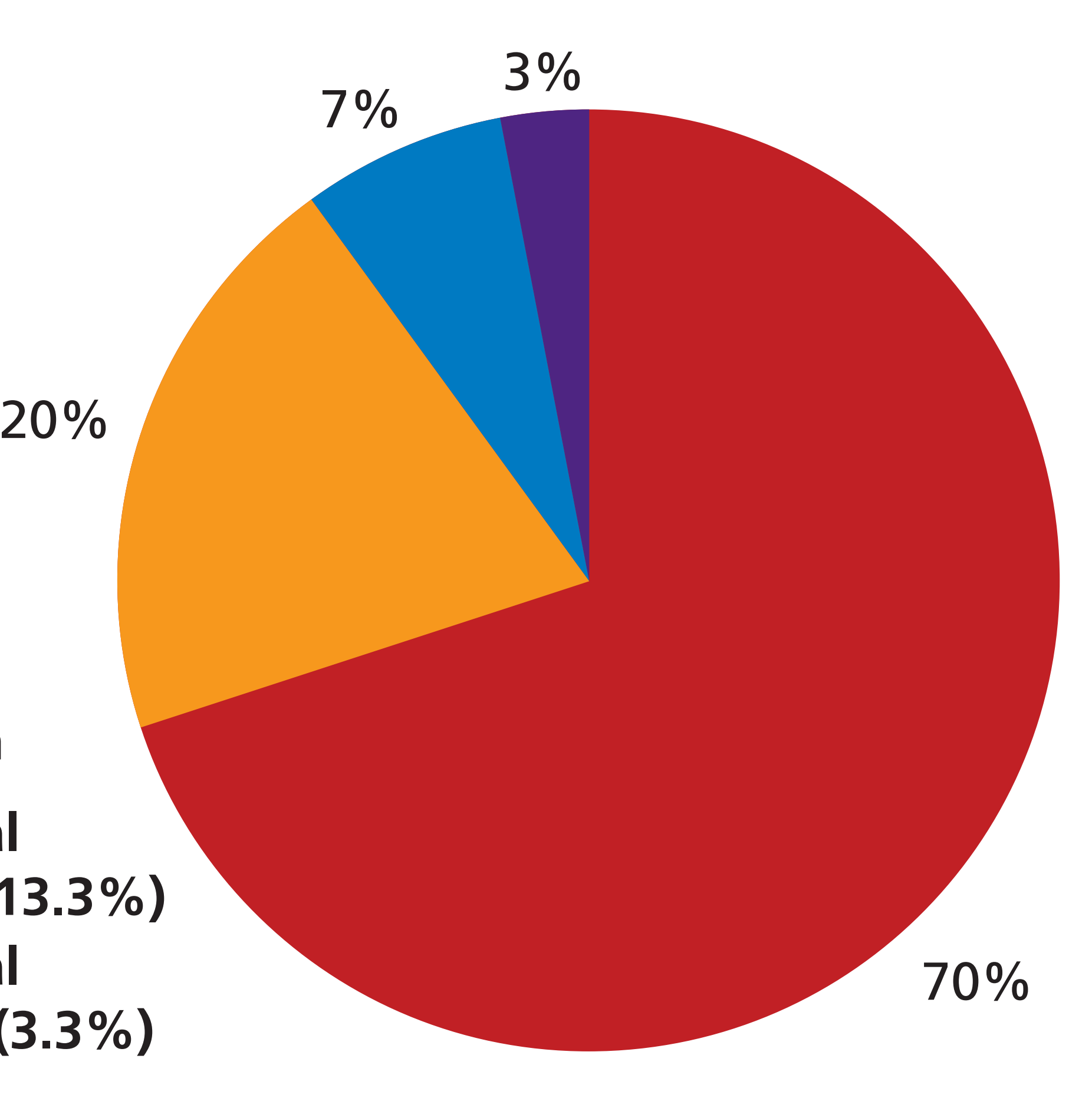

\section{Conclusion}

Primary hypothyroidism is a common adverse effect of TKI therapy. The incidence of primary hypothyroidism in this cohort was $20 \%$ and is similar to hypothyroidism rates in published data from Sunitinib studies (14-46\%). There appeared to be a significant delay between the diagnosis of overt primary hypothyroidism and the start of LT4 therapy. Regular pre-cycle TFT checks and close liaison with Endocrinology will help reduce morbidity from delayed diagnosis and treatment of hypothyroidism. 